\title{
Dielectric Normal Mode Process of Star-Shaped Polyisoprenes
}

\author{
Hirotsugu Yoshida, Keiichiro Adachi, Hiroshi Watanabe, \\ and Tadao KOTAKA* \\ Department of Macromolecular Science, Faculty of Science, \\ Osaka University, Toyonaka, Osaka 560, Japan
}

(Received March 6, 1989)

\begin{abstract}
The dielectric behavior of six-arm star-shaped cis-polyisoprenes (6S-PI) with the arm molecular weight $M_{\mathrm{p}}$ ranging from $3.5 \times 10^{3}$ to $21 \times 10^{3}$ was compared with that of corresponding linear cis-polyisoprenes (L-PI) which were precursors for the arms. In the entangled regime, the normal mode relaxation time $\tau_{\mathrm{n}}$ of 6S-PI was longer and its dependence on molecular weight was stronger for 6S-PIs than for L-PIs. However, an exponential increase of $\tau_{\mathrm{n}}$ with $M_{\mathrm{p}}$ which is characteristic of a highly entangled star chains was not clearly observed for the 6S-PIs examined, because $M_{\mathrm{p}}$ was not large enough. In the non-entangled regime, the relaxation time $\tau_{\mathrm{n} \zeta}$ at an iso-friction state of 6S-PI was close to that of corresponding L-PI, as suggested by the RouseHam theory. However, the relaxation mode distribution for 6S-PI was broader than that of L-PI. A model calculation using Rouse-Ham dynamics suggested that the distribution of arm length could possibly lead to a substantial broadening for the relaxation mode distribution.
\end{abstract}

KEY WORDS Dielectric Relaxation / Normal Mode Process / cisPolyisoprene / Star-Shaped cis-Polyisoprene / Dielectric Relaxation Time / Rouse-Ham Theory /

Dynamics of polymer chains is strongly influenced through their topological structure as well as molecular weight. Among polymers with various architectures such as star- ${ }^{1-5}$ ring-, ${ }^{6,7} \mathrm{H}$-shaped, ${ }^{8}$ and comb-shape, ${ }^{9} \mathrm{dy}$ namics of star-shaped polymers have been most widely studied by means of viscoelastic $^{1-3}$ and diffusion measurements. ${ }^{4,5}$ The Rouse, ${ }^{10}$ Ham, ${ }^{11}$ and Zimm-Kilb ${ }^{12}$ theories were applied to non-entangled star-polymers, whereas the tube model proposed by de Gennes $^{13}$ and developed by Doi and Edwards ${ }^{14}$ for entangled linear polymers was extended to entangled star polymers. ${ }^{15-18}$ These theories for entangled star polymers assume path-breathing (stretching-and-retraction of star arms) to be the dominant relaxation mechanism.

In general, viscoelastic measurements pro-

\footnotetext{
* To whom correspondence should be addressed.
}

vide information on the time evolution of orientation anisotropy of polymer chains, ${ }^{18}$ and have been used extensively for studies of polymer dynamics. For polymer chains having components of dipole moments aligned in the same direction parallel to the chain contour, which are classified as type-A polymers, ${ }^{19}$ fluctuation of end-to-end vectors induces changes in polarization and is dielectrically active. Thus, for such type-A polymers, dielectric measurements are useful to obtain other information for polymer dynamics, an end-to-end vector correlation function. Such a dielectric relaxation process due to a global motion of polymer chains is called a normal mode process. $^{20}$

Stockmayer ${ }^{19}$ first observed dielectric relaxation with characteristic time being dependent on molecular weight for linear 
poly(propylene oxide) (PPO), and attributed it to an end-to-end vector fluctuation. Later, we also found dielectric normal mode processes for some other type-A linear polymers such as cis-polyisoprene (cis-PI). ${ }^{20-23}$

Only a few studies on the dielectric normal mode process have been reported so far branched polymers. ${ }^{24-27}$ Stockmayer and Burke ${ }^{24}$ reported the normal mode process for PPO with three arms; two of $\mathrm{us}^{25}$ for randomly branched poly(2,6-dichloro-1,4-phenylene oxide) (PDCPO) in dilute solution; and Boese et al. $^{26}$ for star-shaped cis-PI with 8 arms. We also reported preliminary dielectric data for star-shaped cis-PI with 6 arms (6S-PI). ${ }^{27}$

For star (or branched) chains, the normal mode process corresponds to fluctuation of end-to-end vectors of the arms (or branches), somewhat different from linear chains. Thus, it is interesting to compare the behavior of star and linear chains. In this paper we present the details of dielectric data on 6S-PIs in bulk and in toluene solution, and compare their relaxation behavior with that of linear cispolyisoprenes (L-PI).

\section{EXPERIMENTAL}

\section{Samples}

Star-shaped cis-PIs with six arms were prepared via anionic polymerization reported by Gervasi and Gosnell. ${ }^{28}$ In each run, first living cis-PI was prepared in heptane with secbutyllithium as the initiator. An aliquot of the living $c i s$-PI was taken and terminated with methanol to recover a precursor L-PI sample, which was used for characterization of the arm of star cis-PI and also for subsequent dielectric measurements. The rest of the living cis-PI was allowed to react with 1,2-bis(trichlorosilyl)ethane for 2 weeks at room temperature. The amount of the coupler was limited to about a half the stoichiometric amount to avoid formation of star-PIs with arms less than six. Then the excess cis-PI was terminated with methanol. The crude product was fractionated from a benzene/methanol mixture to separate $6 \mathrm{~S}-\mathrm{PI}$ and remaining precursor chains. The procedure was essentially the same as in the case of fractionating star-shaped polystyrenes $^{3}$ except that we added $0.02 \%$ of an antioxidant, 2,6-di-tert-butyl-1,4-hydroxytoluene (BHT), to the present fractionation solvents. Three $6 \mathrm{~S}$-PI samples and corresponding L-PI samples were thus prepared.

The weight-average molecular weight $\bar{M}_{w}$ and weight-average to number-average molecular weight ratio $\bar{M}_{w} / \bar{M}_{n}$ were determined on a gel permeation chromatograph (Tosoh HLC-8) equipped with a low-angle lightscattering detector (Tosoh, LS-8000). The characteristics of the samples are summarized in Table $\mathrm{I}$, in which star cis-PI samples are coded as $6\left(\mathrm{I}-M_{\mathrm{p}}\right)$ with the arm molecular weight $M_{\mathrm{p}}$ in the unit of $\mathrm{kg} \mathrm{mol}^{-1}$ and the precursor cis-PIs as I- $M_{\mathrm{p}}$. The arm number $f$ is evaluated by the ratio of the $\bar{M}_{w}$ of the star- to the linear (precursor) cis-PIs.

The solvent toluene for dielectric measurements was dried with calcium hydride and then distilled under vacuum at room temperature just before use.

Table I. Characteristics of star-shaped PI and linear cis-PI

\begin{tabular}{|c|c|c|c|c|c|c|}
\hline \multicolumn{4}{|c|}{ Star-PI } & \multicolumn{3}{|c|}{ Precursor PI } \\
\hline Code & $10^{-3} \bar{M}_{w}$ & $\bar{M}_{w} / \bar{M}_{n}$ & $f$ & Code & $10^{-3} \bar{M}_{w}$ & $\bar{M}_{w} / \bar{M}_{n}$ \\
\hline $6(\mathrm{I}-04)$ & 20.2 & 1.08 & 5.8 & $\mathrm{I}-04$ & 3.50 & 1.07 \\
\hline $6(I-09)$ & 54.2 & 1.05 & 5.7 & I-09 & 9.49 & 1.07 \\
\hline $6(\mathrm{I}-21)$ & 117 & 1.06 & 5.7 & I-21 & 20.7 & 1.05 \\
\hline
\end{tabular}




\section{Method}

Dielectric measurements were carried out with a transformer bridge (GR-1615A) at $210-370 \mathrm{~K}$ for bulk and $185-273 \mathrm{~K}$ for toluene solutions of PIs. The range of frequency examined was from 0.02 to $50 \mathrm{kHz}$. The details of the dielectric cell were described previously. ${ }^{21}$ Since the frequency range available was limited, the time-temperature superposition principle ${ }^{29}$ was used to obtain master curves for complex dielectric constants $\varepsilon^{*}$. The shift factor $a_{\mathrm{T}}$ of the star cis-PIs was the same as that of linear cis-PIs in both bulk and toluene solutions. ${ }^{21,23}$

\section{RESULTS AND DISCUSSION}

Dielectric Loss Curves of Star-Shaped and Linear cis-PI

Figure 1 shows the frequency dependence of the dielectric loss factor $\varepsilon^{\prime \prime}$ reduced at $273 \mathrm{~K}$ for three bulk $6 \mathrm{~S}$-PIs and the corresponding precursor L-PIs. The loss peaks seen below $100 \mathrm{kHz}$ are due to the normal mode process, while those around $10 \mathrm{MHz}$ are due to the segmental mode process. The loss maximum frequencies $f_{\mathrm{mn}}$ of the normal mode process depend strongly on the molecular weight, while those $f_{\mathrm{ms}}$ of the segmental mode do not, as found previously. ${ }^{20,21}$ Comparing the star and corresponding linear $c i s$-PI, we see that $f_{\mathrm{mn}}$ is lower and the shape of the loss curve is broader for star PI. In contrast to these differences in the normal mode process, the segmental mode process is independent of the architecture of the cis-PI molecules.

\section{Molecular Weight Dependence of the Normal Mode Relaxation Time}

From the peak frequencies $f_{\mathrm{mn}}$ and $f_{\mathrm{ms}}$, respectively, for the normal and segmental mode processes, the characteristic times are evaluated by

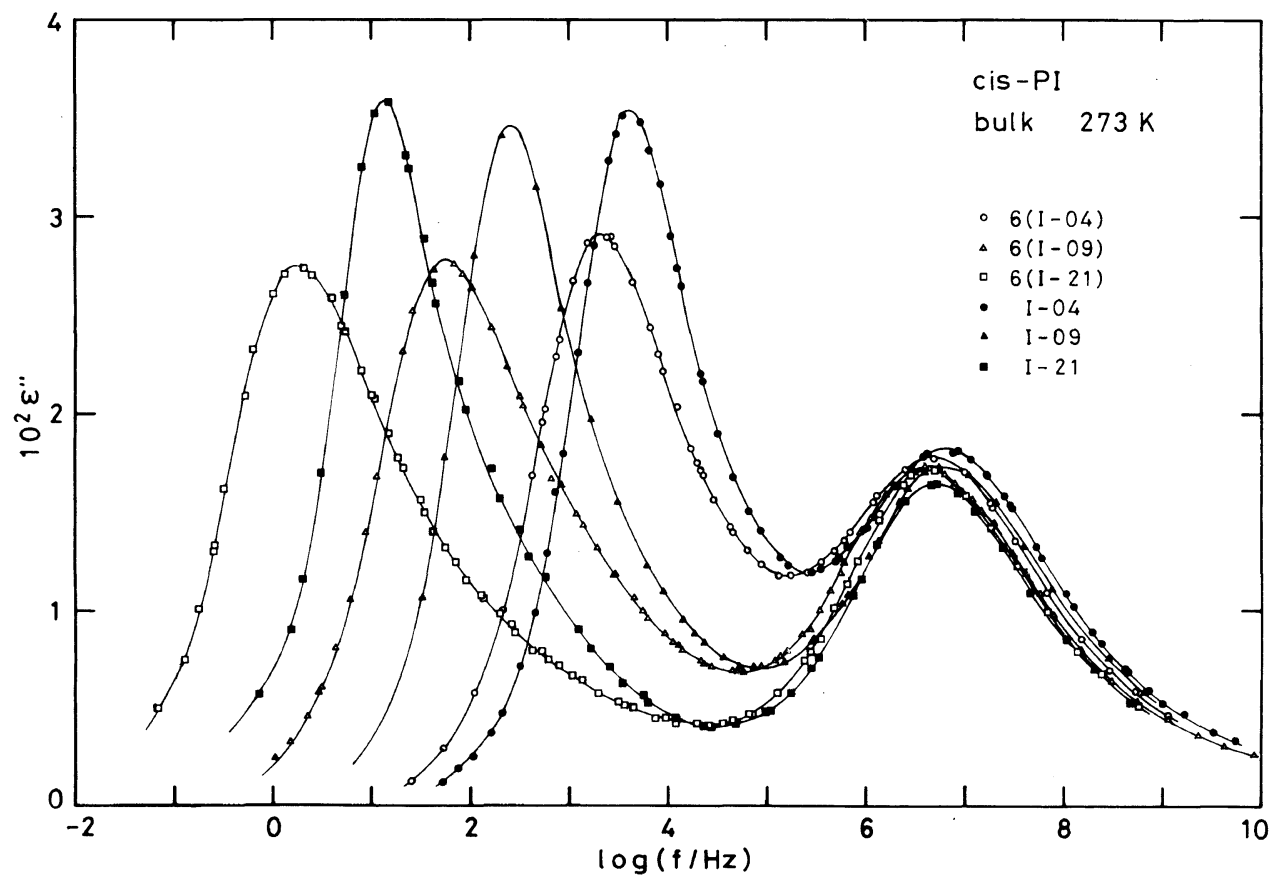

Figure 1. Master curves of the frequency dependence of $\varepsilon^{\prime \prime}$ for six-arm star-shaped and linear precursor cis-PIs at $273 \mathrm{~K}$ in the bulk state. The precursor cis-PIs have the same molecular weight as the arm of the corresponding star-shaped cis-PI. 


$$
\tau_{\mathrm{n}}=1 /\left(2 \pi f_{\mathrm{mn}}\right), \quad \tau_{\mathrm{s}}=1 /\left(2 \pi f_{\mathrm{ms}}\right)
$$

where $\tau_{\mathrm{n}}$ and $\tau_{\mathrm{s}}$ are certain average relaxation times for the respective processes, and are determined by the distribution of the relaxation modes. In particular, for narrow modedistribution which leads to a sharp $\varepsilon^{\prime \prime}$ curve, $\tau$ given by eq 1 is close to the longest relaxation time of that process. On the other hand, for broad mode-distribution such as observed for the normal mode process of 6S-PIs, $\tau$ given by eq 1 should be substantially shorter than the longest relaxation time.

Figure 2 shows the molecular weight dependence of $\tau_{\mathrm{n}}$ and $\tau_{\mathrm{s}}$ for the star- and linear cis-PIs in the bulk state at $273 \mathrm{~K}$. For star cisPIs, $\tau_{\mathrm{n}}$ and $\tau_{\mathrm{s}}$ are plotted against the arm molecular weight $M_{\mathrm{p}}$. The data for 8-arm cisPIs reported by Boese et al. ${ }^{26}$ are also plotted in this figure.

Because of the effect of the chain ends on the free volume fractions, ${ }^{29}$ the segmental friction coefficient $\zeta$ decreases with decreasing molecular weight. Thus, in Figure 2, correction for reducing $\tau_{\mathrm{n}}$ and $\tau_{\mathrm{s}}$ to an iso- $\zeta$ state is necessary for those of I-04. Assuming that $\tau_{\mathrm{s}}$ is independent of molecular weight at an iso- $\zeta$ state, we reduced the $\tau_{\mathrm{s}}$ and $\tau_{\mathrm{n}}$ of I-04 to the iso- $\zeta$ state for the longer PIs, as indicated by the dashed lines in Figure 2. We note that $\tau_{\mathrm{n}}$ of $6(\mathrm{I}-04)$ is nearly the same as that of the I-04 sample thus corrected.

The arm molecular weight $M_{\mathrm{p}}(=3500)$ of the 6(I-04) sample is smaller than the entanglement spacing $M_{\mathrm{e}}(\simeq 5000)$ for bulk PI. Thus, the 6(I-04) sample appears to be in the nonentangled regime where the Rouse-Ham theory ${ }^{10-12}$ can be applied. As discussed elsewhere in greater detail, ${ }^{30}$ this theory predicts monodisperse star chains with all the identical arm-length $M_{\mathrm{p}}$ to have $\tau_{\mathrm{n}}$ being exactly the same as that of the linear chains with $M=M_{\mathrm{p}}$. This is in accordance with the observation in Figure 2.

In Figure 2, we also note that for 6S-PIs in the entangled regime $\left(M_{\mathrm{p}}>M_{\mathrm{e}}\right) \tau_{\mathrm{n}}$ is longer

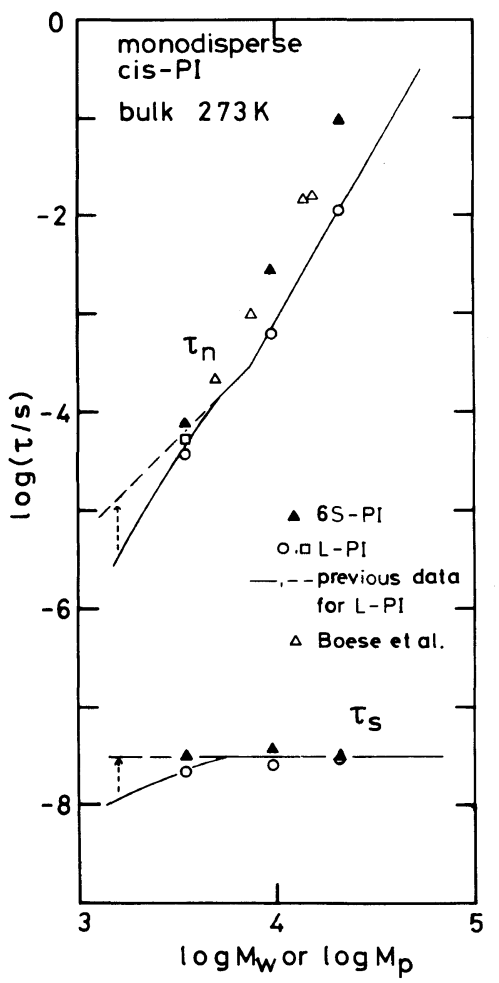

Figure 2. Molecular weight dependence of the relaxation time $\tau_{\mathrm{n}}$ for the normal mode process and that of $\tau_{\mathrm{s}}$ for the segmental mode process at $273 \mathrm{~K}$ for $6 \mathrm{~S}-\mathrm{PI}(\boldsymbol{\Delta})$ and L-PI $(\mathrm{O})$. The abscissa indicates the arm molecular weight $M_{\mathrm{p}}$ for $6 \mathrm{~S}-\mathrm{PIs}$ and the overall molecular weight for L-PIs. The dashed lines represent the relaxation times of L-PI reduced at an iso-friction state. The $\tau_{\mathrm{n}}$ for I-04 reduced to the iso-friction state is plotted by squares. The open triangles indicate the data for 8S-PI reported by Boese et al. ${ }^{26}$

Table II. Relaxation times for normal mode processes of 6S-PIs and L-PIs

\begin{tabular}{|c|c|c|c|}
\hline \multirow{2}{*}{$M_{\mathrm{p}}$} & \multicolumn{2}{|c|}{$\log \tau_{\mathbf{n}} / s^{\mathbf{a}}$} & \multirow{2}{*}{$\log \left(\tau_{\mathrm{n}}{ }^{6 \mathrm{~S}-\mathrm{PI}} / \tau_{\mathrm{n}}{ }^{\mathrm{L}-\mathrm{PI}}\right)$} \\
\hline & 6S-PI & L-PI & \\
\hline 3500 & -4.12 & $-4.27^{b}$ & 0.15 \\
\hline 9490 & -2.56 & -3.20 & 0.64 \\
\hline 20700 & -1.02 & -1.96 & 0.94 \\
\hline
\end{tabular}

a Determined from peak frequencies with eq 1 .

${ }^{b}$ Reduced to the iso-friction state.

and its molecular weight dependence is somewhat stronger than that for L-PIs (exhibiting a power-law relation $\tau_{n} \propto M^{3.5}$ ). This tendency 
can be more clearly observed in Table II, where the ratios $\tau_{\mathrm{n}}{ }^{6 \mathrm{~S}-\mathrm{PI}} / \tau_{\mathrm{n}}{ }^{\mathrm{L}-\mathrm{PI}}$ are summarized. However, it is not straightforward to estimate the exact molecular-weight dependence of the longest relaxation time $T_{\max }$ for $6 \mathrm{~S}-\mathrm{PIs}$ in the entagled regime. Because the shape of $\varepsilon^{\prime \prime}$ curves, and hence the relaxation-mode distribution changes a little with increasing molecular weight ( $c f$., Figure 4 ), the $T_{\max } / \tau_{\mathrm{n}}$ ratio changes accordingly. Note that $\tau_{\mathrm{n}}$ is not necessary in agreement with $T_{\max }$.

For highly entangled star-shaped polymers, theories ${ }^{15-17}$ assuming the path-breathing mechanism predict $T_{\max }$ to be scaled as

$$
T_{\max } \propto \zeta M_{\mathrm{p}}{ }^{\alpha} \exp \left(v M_{\mathrm{p}} / M_{\mathrm{e}}\right)
$$

where the constants $\alpha$ and $v$ are evaluated to be 3 and 0.66 by Doi and Kuzuu, ${ }^{16}$ and 1.5 and 0.6 by Pearson and Helfand. ${ }^{17}$ All these theories predict that $T_{\max }$ (as well as viscosity) increases exponentially with increasing $M_{\mathrm{p}}$ $\left(\gg M_{\mathrm{e}}\right.$ ). This behavior is called exponential enhancement, and has been observed experimentally for viscoelastic quantities. ${ }^{2}$ However, the 6S-PIs examined in this study did not clearly exhibit the exponential increase of $\tau_{n}$ with $M_{\mathrm{p}}$, most probably because $M_{\mathrm{p}}$ was not large enough to enter the highly entangled regime as demanded by the theory. The two star samples, 6(I-09) and 6(I-21), appear to be in a lightly to modestly entangled regime, where $\tau_{\mathrm{n}}$ is longer and its $M$ dependence is somewhat stronger for stars as compared to the corresponding linear chains.

\section{Dielectric Relaxation Mode Distribution}

Figure 3 compares the normalized master curves of $\varepsilon^{\prime \prime}$ for toluene solutions of 6(I-21) and I-21. The difference in the broadness of the loss curves between the 6S- and L-PI samples is similar to that in the bulk. We also note that, for the L-PI sample, the shape of the $\varepsilon^{\prime \prime}$ curve remains the same at high concentration $\left(c \geqq 55 \mathrm{wt}^{\circ} \%\right.$ ), and is slightly narrower at low $c$ ( $\leqq 25 \mathrm{wt} \%$ ). For the 6S-PI sample, the change in the shape with $c$ appears to be more prominent as compared to the L-PI sample.

Figure 4 compares the normalized $\varepsilon^{\prime \prime}$ curves of 6S-PIs and L-PIs in the bulk state. The increase in $\varepsilon^{\prime \prime}$ at high frequencies is due to the onset of the segmental mode process. We see that for 6S-PIs, the $\varepsilon^{\prime \prime}$ curves become a little broader with increasing $M_{\mathrm{p}}$, while for L-PIs this trend is not prominent. The dashed curve in Figure $4 \mathrm{~A}$ indicates the $\varepsilon^{\prime \prime}$ curve for L-PIs. Again, we confirm the shape of the loss curve, i.e., the relaxation-mode distribution of $6 \mathrm{~S}$-PIs is broader than that of L-PIs.

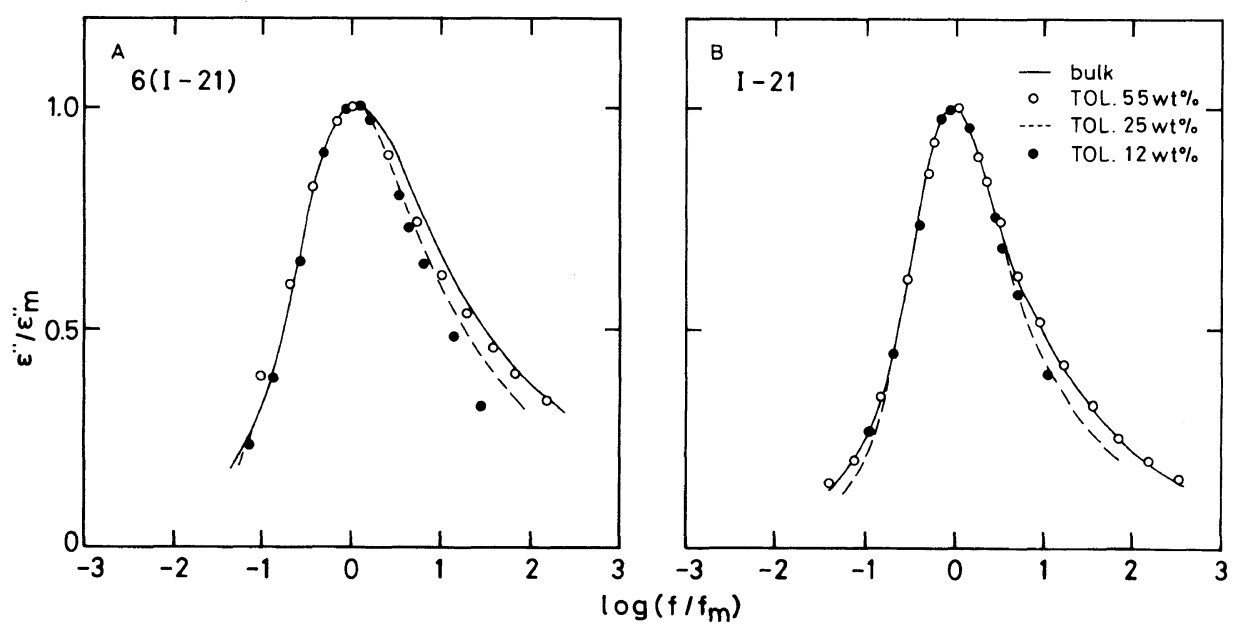

Figure 3. Normalized master curves of $\varepsilon^{\prime \prime}$ for toluene solutions of 6(I-21) (A) and I-21 (B) at concentrations of $12,25,55$, and $100 \mathrm{wt} \%$. 

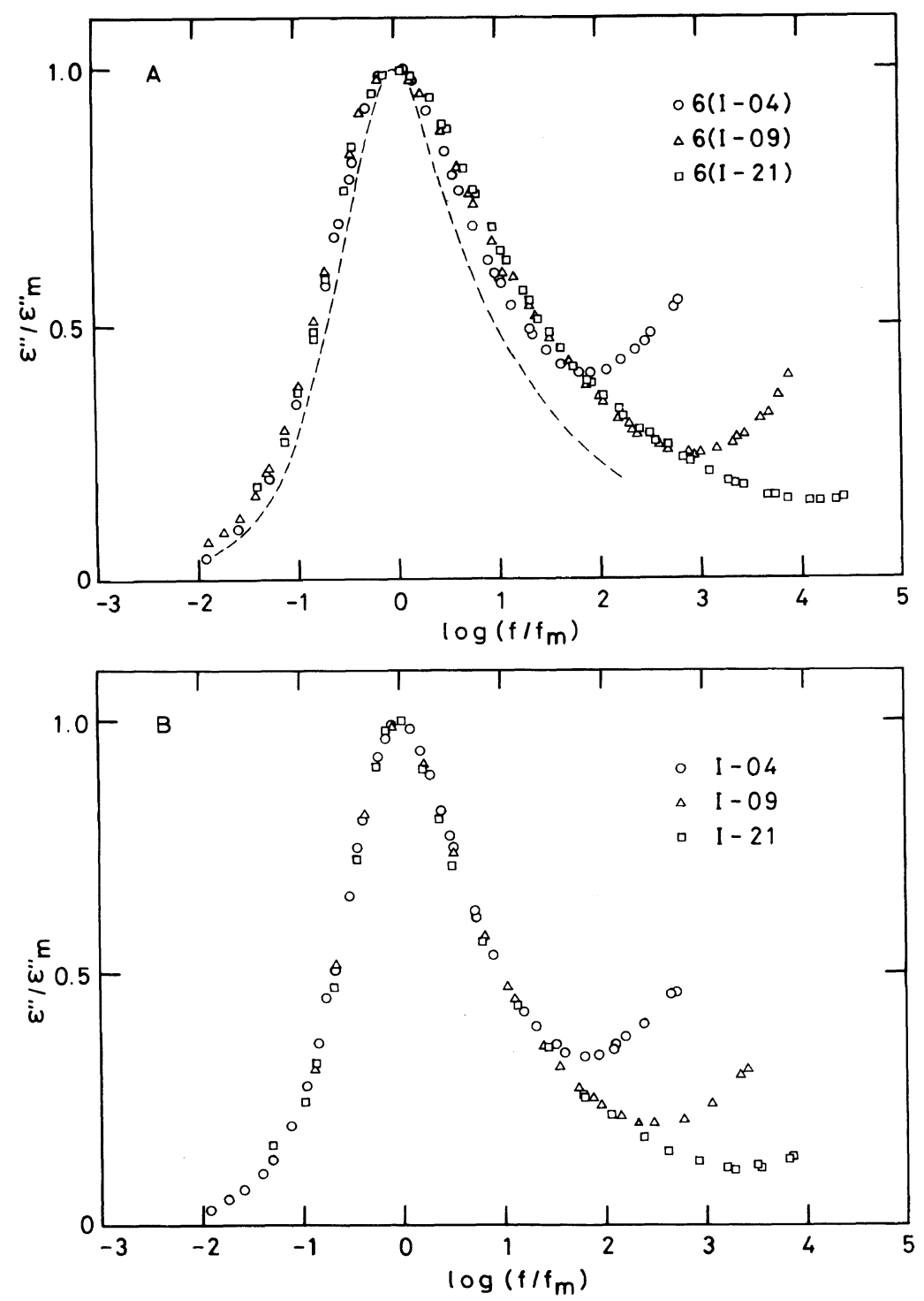

Figure 4. Comparison of the shapes of the $\varepsilon^{\prime \prime}$ curves for (A) 6S-PIs and (B) L-PIs at bulk state. The $\varepsilon^{\prime \prime}$ curve for the L-PIs is indicated in Figure $4 \mathrm{~A}$ by the dashed curve for easy comparison.

This feature can be a little more clearly chains calculated from the $\varepsilon^{\prime \prime}$ data by the observed in dielectric relaxation spectra $g(\tau)$ defined by method reported previously. ${ }^{22}$ In this method, $g(\tau)$ is given by the sum of delta functions

$$
\varepsilon^{\prime \prime}=\int_{-\infty}^{\infty} \frac{\omega \tau}{1+\omega^{2} \tau^{2}} g(\tau) \mathrm{d} \ln \tau
$$

Figure 5 demonstrates $g(\tau)$ of the star (6(I-21); parts A and B) and linear (I-21; parts C and D)

$$
\begin{aligned}
g(\tau) & =\sum_{p} g_{p} \delta\left(\tau-\tau_{p}\right), \quad \tau_{p}=\text { const. } \Delta^{p} \\
p & =0, \pm 1, \pm 2, \cdots
\end{aligned}
$$




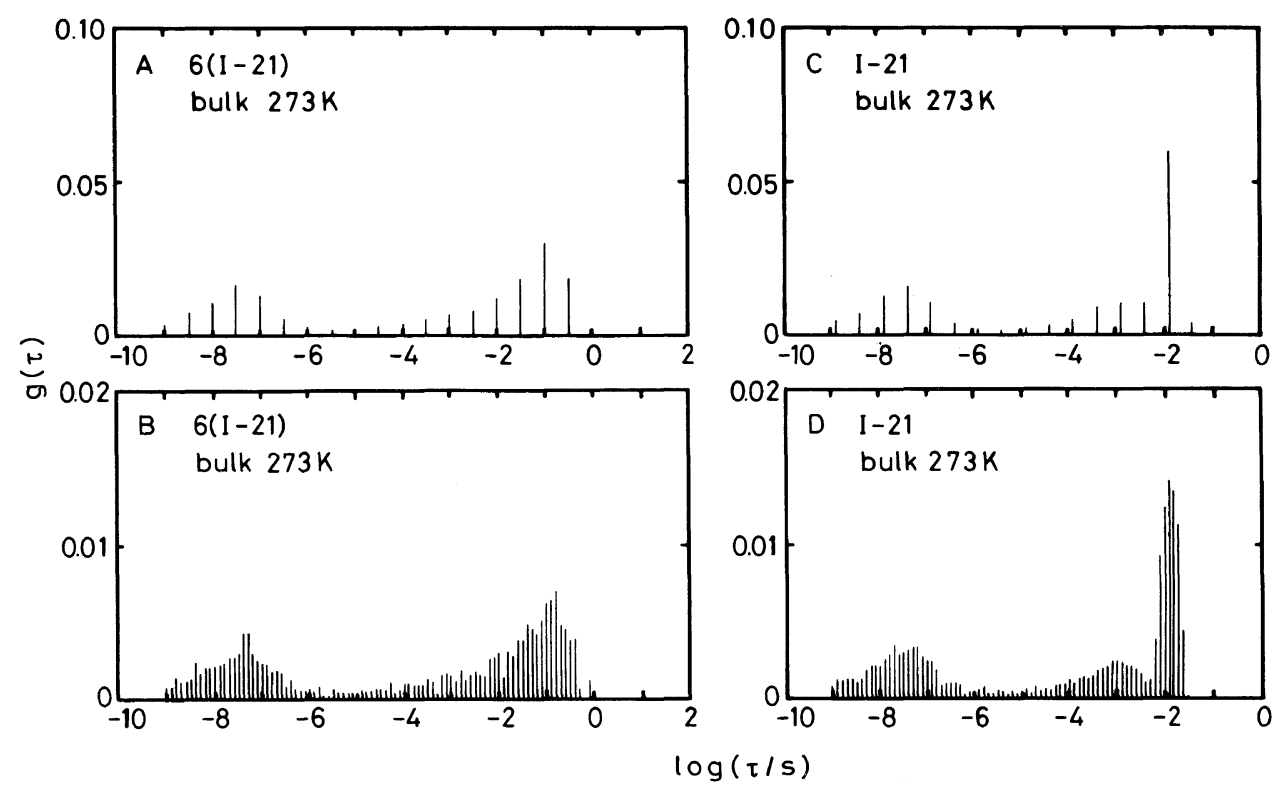

Figure 5. Dielectric relaxation spectra for $6(\mathrm{I}-21)(\mathrm{A}, \mathrm{B})$ and $\mathrm{I}-21(\mathrm{C}, \mathrm{D})$ evaluated from the $\varepsilon^{\prime \prime} \mathrm{data}$. The $g(\tau)$ was assumed to be the sum of delta functions $g_{\mathrm{p}} \delta\left(\tau-\right.$ const. $\left.\Delta^{\mathrm{p}}\right): \Delta$ was chosen to be $10^{0.5}$ (A and C) and $10^{0.1}$ (B and D).

and the difference between the $\varepsilon^{\prime \prime}$ data and calculated $\varepsilon^{\prime \prime}$ (eq 3 ) is minimized to determine the intensity factors $g_{p}$. The parts $\mathrm{A}$ and $\mathrm{C}$ in Figure 5 are the spectra obtained for $\Delta=10^{0.5}$ for star and linear PI's, respectively, and the parts $\mathrm{B}$ and $\mathrm{D}$ those for $\Delta=10^{0.1}$. The $\varepsilon^{\prime \prime}$ data do not allow us to determine the most appropriate $\tau_{p}$ values ( $\Delta$ values) uniquely, as demonstrated in Figure 6. The two spectra shown in Figures 5C and 5D give indistinguishable $\varepsilon^{\prime \prime}$ curves which reproduce the $\varepsilon^{\prime \prime}$ data within experimental uncertainty. However, the general feature of the spectra deduced from Figure 5 is enough to demonstrate the difference in $g(\tau)$ of star and linear chains. In both Figures $5 \mathrm{C}$ and 5D, we note that the relaxation intensity for L-chain is much higher in the longest time region than in the shorter time region. On the other hand, Figures $5 \mathrm{~A}$ and $5 \mathrm{~B}$ clearly indicate that substantial relaxation intensity exists even at an intermediate to short time region. These features are seen also for samples with lower molecular weight and are irrespective of the entanglement effect. Again, we see the relaxation-mode distribution of the normal mode process for 6S-PIs is broader than that for L-PIs.

The broad relaxation-mode distribution for the 6S-PIs examined may partly be due to differences between the dynamics of star and linear chains (at around crossover molecular weight $M_{\mathrm{p}} \simeq M_{\mathrm{e}}$ ), and may also be due to distribution of the arm molecular weight $M_{\mathrm{p}}$. Here, we briefly examine the effects of the $M_{\mathrm{p}}$ distribution on $\varepsilon^{\prime \prime}$ curves for star chains in the non-entangled regime, where Rouse-Ham dynamics are well established and the dielectric response can be calculated accordingly.

As described elsewhere in greater detail, ${ }^{30}$ the $\varepsilon^{\prime \prime}$ curve of star (Rouse-Ham) chains having monodisperse $f$ arms composed of $N$ segments is given by

$$
\varepsilon_{\mathrm{S}, \mathrm{m}}^{\prime \prime}(\omega, N)=Q v_{\mathrm{a}} N b^{2} \sum_{p=\text { odd }} \frac{8}{p^{2} \pi^{2}} \frac{\omega \tau_{p}(N)}{1+\omega^{2} \tau_{p}(N)^{2}}
$$

where $v_{\mathrm{a}}(\propto c / N)$ is the number of arms per 


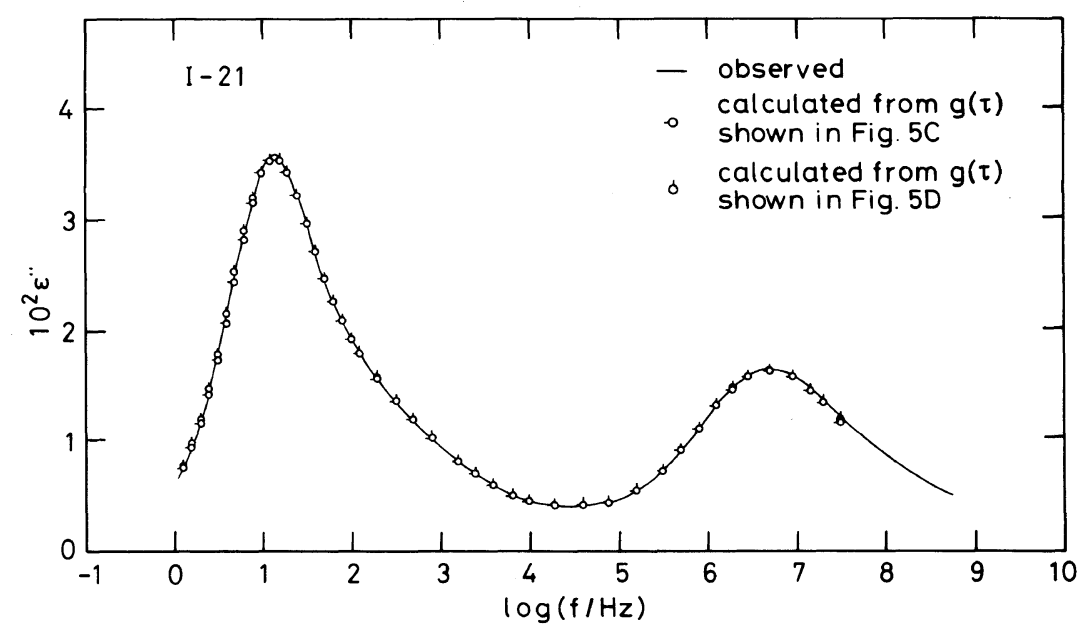

Figure 6. Comparison of the observed $\varepsilon^{\prime \prime}$ curve $(-)$ and those calculated from the two dielectric relaxation spectra $(-\bigcirc$; from $g(\tau)$ given in Figure 5C, $O$; from Figure 5D) for I-21.

unit volume, $b$ is the segment step-length, $Q$ is a constant related to the magnitude of dipole moment per segment, and $\tau_{p}(N)$ is the relaxation time for the dielectrically active $p$-th mode given by

$$
\tau_{p}(N)=\zeta N^{2} b^{2} / 3 \pi^{2} k T p^{2} \quad \text { for } \quad N \gg 1
$$

with $k T$ being the thermal energy. Clearly, $\varepsilon_{\mathrm{S}, \mathrm{m}}^{\prime \prime}$ is exactly the same as that for monodisperse linear chains each composed of $N$ segments, i.e., those identical to the arms. This result has been obtained because of a high symmetry of the star-chain architecture and resulting degeneracy of Rouse-Ham eigenfunctions. ${ }^{30}$ Obviously, eq 5 cannot explain the data shown in Figure 4.

If all arms in a star chain do not have the same molecular weight, the symmetry of star architecture disappears, and the eigenmode distribution becomes broader. To examine this point in a qualitative way, we compared the $\varepsilon$ " curves of two model 6-arm stars, one having equal arms each composed of $5 N / 3$ segments and the other having four $2 \mathrm{~N}$-segments arms and two $N$-segments arms. Both of the two stars have the same molecular weight $10 N$ (and are monodisperse), and the only difference is arm-length distribution.
Figure 7 compares the shape of the $\varepsilon^{\prime \prime}$ curves for these two model star chains with equal arms and bimodal arm-length distribution (with $\bar{M}_{w}{ }^{\text {arm }} / \bar{M}_{n}{ }^{\text {arm }}=1.08$ ). The solid and dashed arrows indicate characteristic frequencies corresponding to the longest relaxation times

$$
T_{\text {max }, \mathrm{m}}=25 \zeta N^{2} b^{2} / 27 \pi^{2} k T
$$

for the former, and

$$
\begin{aligned}
& T_{\max , \mathrm{b}}=\zeta b^{2} N^{2} / 3 \theta^{2} k T, \\
& \theta=\cos ^{-1} \sqrt{1 / 6}, \quad 0<\theta<\pi / 2
\end{aligned}
$$

for the latter. ${ }^{30}$

In Figure 7, we note that the peak frequencies for the two cases are nearly the same, despite the fact that $T_{\max }$ (arrows) are significantly different. Clearly, the $\varepsilon^{\prime \prime}$ curve is substantially broader for the star chain with armlength distribution. This feature can be more clearly observed in Figure 8, where the relaxation spectra obtained from the RouseHam theory are compared. Note again that the $\varepsilon^{\prime \prime}$ curve (and the spectrum) of the star with equal-arms is identical to that of the monodisperse linear chain composed of $5 N / 3$ segments.

The results in Figures 7 and 8 suggest that 


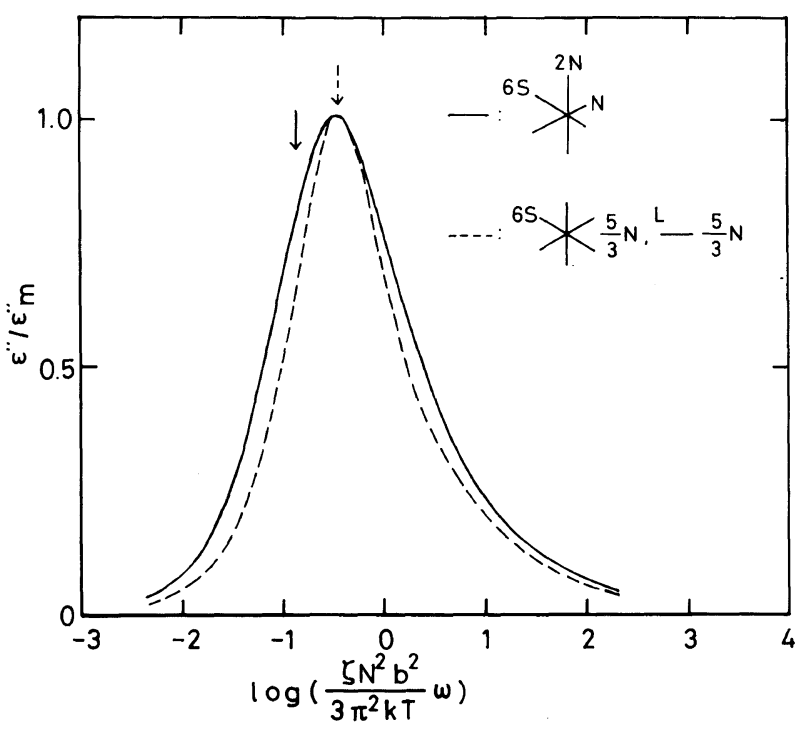

Figure 7. Comparison of the $\varepsilon^{\prime \prime}$ curves of two model star chains having the same molecular weight: a star chain with four arms with length $2 N$ and two arms with length $N$; ---, a star chain with six arms with length $5 N / 3$. The solid and dashed arrows in the figure indicate the characteristic frequencies for the slowest mode for the former and the latter, respectively.

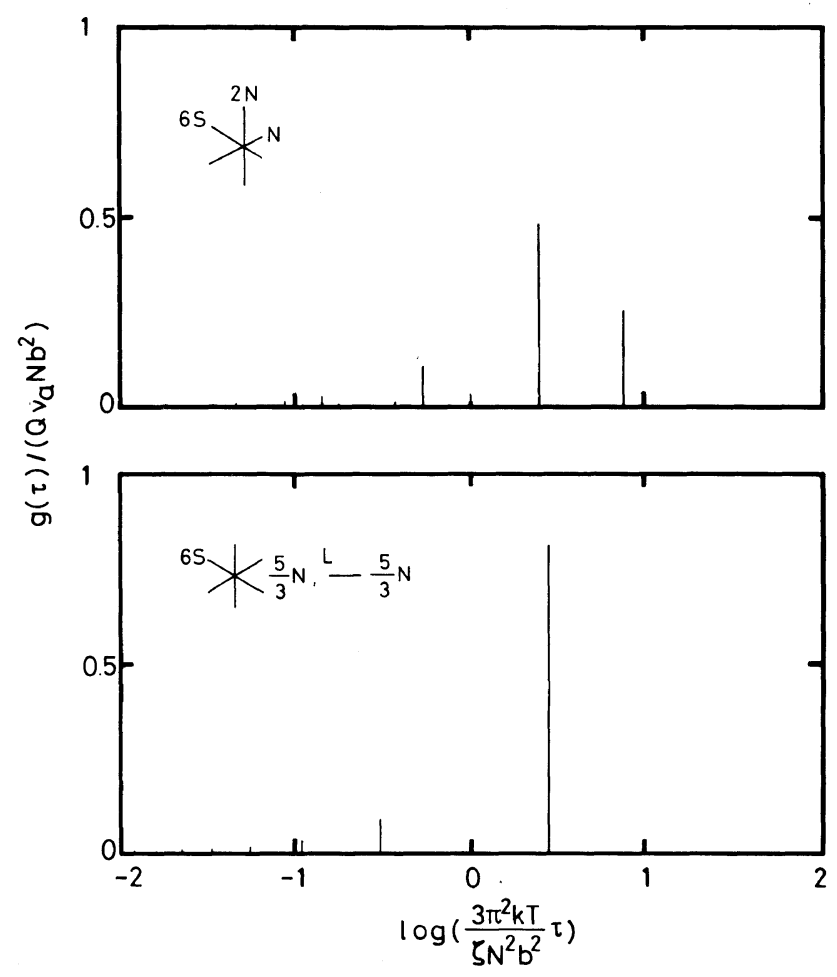

Figure 8. Comparison of the spectra for the two model star chains examined in Figure 7. 
arm length distribution remarkably broadens the $\varepsilon$ " curve of the star polymers. In addition, the molecular weight distribution of star chains themselves also leads to a broader $\varepsilon^{\prime \prime}$ curve. This second effect may be the same for star and linear chains in the non-entangled regime, but larger for star chains in the entangled regime because of the stronger $M$ dependence of $T_{\max }$. From these points, the difference in the $\varepsilon$ " curves observed in Figure 4 can be explained qualitatively by considering primarily arm-length distribution. Detailed analysis and explanation for that difference are a challenging problem, and we consider this elsewhere ${ }^{30}$ in greater detail.

Acknowledgements. This work was supported in part by a Grant-in-Aid for Scientific Research (No. 6055062) from the Ministry of Education, Science, and Culture of Japan and also support from the Institute of Macromolecular Research (Osaka University) is also gratefully acknowledged.

\section{REFERENCES}

1. T. Masuda, Y. Ohta, and S. Onogi, Macromolecules, 4, 763 (1971).

2. W. W. Graessley, T. Masuda, J. Roovers, and N. Hadjichristidis, Macromolecules, 9, 127 (1976).

3. H. Watanabe, H. Yoshida, and T. Kotaka, Macromolecules, 21, 2175 (1988) and papers cited.

4. M. Antonietti and H. Sillescu, Macromolecules, 19, 798 (1986).

5. K. R. Shull, E. J. Kramer, G. Hadziioannou, M. Antonnietti, and H. Sillescu, Macromolecules, 21, 2578 (1988).

6. J. Roovers, Macromolecules, 18, 1359 (1985); ibid., 21, 1517 (1988).
7. G. B. McKenna, G. Hadziioannou, P. Lutz, G. Hild, C. Strazielle, C. Straupe, P. Rempp, and A. J. Kovacs, Macromolecules, 20, 498 (1987).

8. J. Roovers, Macromolecules, 17, 1196 (1984).

9. T. Fujimoto, H. Kajiwara, M. Hirose, and $\mathbf{M}$. Nagasawa, Polym. J., 3, 181 (1972).

10. P. E. Rouse, J. Chem. Phys., 21, 1272 (1953).

11. J. S. Ham, J. Chem. Phys., 26, 625 (1957).

12. B. H. Zimm and R. W. Kilb, J. Polym. Sci., 37, 294 (1959).

13. P. G. de Gennes, J. Chem. Phys., 55, 572 (1971).

14. M. Doi and S. F. Edwards, J. Chem. Soc., Faraday Trans. 2, 74, 1789 (1978); see, also, M. Doi and S. F. Edwards, "The Theory of Polymer Dynamics," Clarendon Press, Oxford, 1986.

15. P. G. de Gennes, J. Phys. (Paris), 36, 1199 (1975).

16. M. Doi and N. Kuzuu, J. Polym. Sci., Polym. Lett. Ed., 18, 775 (1980).

17. D. S. Pearson and E. Helfand, Macromolecules, 17, 888 (1984).

18. see, for example, W. W. Graessley, Adv. Polym. Sci., 47, 67 (1982)

19. W. H. Stockmayer, Pure Appl. Chem., 15, 539 (1969).

20. K. Adachi and T. Kotaka, Macromolecules, 18, 466 (1985).

21. Y. Imanishi, K. Adachi, and T. Kotaka, J. Chem. Phys., 89, 7585 (1988).

22. Y. Imanishi, K. Adachi, and T. Kotaka, J. Chem. Phys., 89, 7593 (1988).

23. K. Adachi, Y. Imanishi, and T. Kotaka, J. Chem. Soc., Faraday Trans. 1, 85, 1065 (1989).

24. W. H. Stockmayer and J. J. Burke, Macromolecules, 2, 647 (1969).

25. K. Adachi and T. Kotaka, Macromolecules, 16, 1936 (1983).

26. D. Boese, F. Kremer, and L. J. Fetters, Makromol. Chem., Rapid Commun., 9, 367 (1988).

27. H. Yoshida, K. Adachi, H. Watanabe, and T. Kotaka, Polym. Prepr. Jpn., 37, 2076 (1988).

28. J. A. Gervasi and A. B. Gosnell, J. Polym. Sci., Polym. Chem. Ed., 4, 1391 (1966).

29. See, for example, J. D. Ferry, "Viscoelastic Properties of Polymers," 3rd ed, Wiley, New York, N.Y., 1980.

30. H. Watanabe, H. Yoshida, and T. Kotaka, Polym. $J$, to be published. 